\title{
Preliminary classification criteria for the cryoglobulinaemic vasculitis
}

\author{
S De Vita, ${ }^{1}$ F Soldano, ${ }^{2} \mathrm{M}$ Isola, ${ }^{2} \mathrm{G}$ Monti, ${ }^{3}$ A Gabrielli, ${ }^{4}$ A Tzioufas, ${ }^{5}$ C Ferri, ${ }^{6}$ \\ G F Ferraccioli, ${ }^{7}$ L Quartuccio, ${ }^{1}$ L Corazza, ${ }^{1}$ G De Marchi, ${ }^{1}$ M Ramos Casals, ${ }^{8}$ \\ M Voulgarelis, ${ }^{9}$ M Lenzi, ${ }^{10} \mathrm{~F}$ Saccardo, ${ }^{3}$ P Fraticelli, ${ }^{4}$ M T Mascia, ${ }^{6}$ D Sansonno, \\ P Cacoub, ${ }^{12}$ M Tomsic, ${ }^{13}$ A Tavoni, ${ }^{14}$ M Pietrogrande, ${ }^{15}$ A L Zignego,${ }^{16}$ S Scarpato, ${ }^{17}$ \\ C Mazzaro, ${ }^{18}$ P Pioltelli, ${ }^{19}$ S Steinfeld, ${ }^{20} \mathrm{P}$ Lamprecht, ${ }^{21} \mathrm{~S}$ Bombardieri, ${ }^{14} \mathrm{M}$ Galli ${ }^{22}$
}

For numbered affiliations see end of article

\section{Correspondence to} Professor S De Vita, Rheumatology Clinic, Department of Medical and Biological Sciences, Azienda Ospedaliero-Universitaria

S Maria della Misericordia, Piazzale Santa Maria

Misericordia 1, 33100 Udine, Italy;

devita.salvatore@aoud.sanita. fvg.it

Accepted 1 April 2011 Published Online First 13 May 2011

\begin{abstract}
Background To develop preliminary classification criteria for the cryoglobulinaemic syndrome or cryoglobulinaemic vasculitis (CV).

Methods Study part I developed a questionnaire for CV to be included in the formal, second part (study part II). Positivity of serum cryoglobulins was defined by experts as an essential condition for CV classification. In study part II, a core set of classification items (questionnaire, clinical and laboratory items, as agreed) was tested in three groups of patients and controls - that is, group A (new patients with the CV), group B (controls with serum cryoglobulins but lacking CV) and group C (controls without serum cryoglobulins but with features which can be observed in CV).

Results In study part I (188 cases, 284 controls), a positive response to at least two of three selected questions showed a sensitivity of $81.9 \%$ and a specificity of $83.5 \%$ for CV. This questionnaire was employed and validated in study part II, which included 272 patients in group $A$ and 228 controls in group B. The final classification criteria for $\mathrm{CV}$, by pooling data from group $A$ and group $B$, required the positivity of questionnaire plus clinical, questionnaire plus laboratory, or clinical plus laboratory items, or all the three, providing a sensitivity of $88.5 \%$ and a specificity of $93.6 \%$ for CV. By comparing data in group A versus group C (425 controls), the same classification criteria showed a sensitivity $88.5 \%$ and a specificity $97.0 \%$ for CV.
\end{abstract}

Conclusion Classification criteria for CV were developed, and now need validation.

Cryoglobulinaemic syndrome or cryoglobulinaemic vasculitis (CV) is a systemic vasculitis associated with serum positive cryoglobulins- that is, immune complexes composed of rheumatoid factor (RF) monoclonal or polyclonal against polyclonal IgG (type II or type III cryoglobulins, respectively) or immunoglobulins without RF activity (type I), which reversibly precipitate or form a gel at a temperature below $37^{\circ} \mathrm{C} .{ }^{12} \mathrm{CV}$ is usually linked to non-malignant B-cell lymphoproliferation, ${ }^{3}$ often triggered by chronic hepatitis $\mathrm{C}$ virus (HCV) infection. ${ }^{45}$

Classification criteria developed with an accepted methodology are presently lacking for CV, while a correct classification is a key step for clinical practice, research and epidemiological studies. ${ }^{6-14}$ Previous criteria were not universal and lacked appropriate statistical support. ${ }^{9-12}$ This study was therefore started, involving different
European experts. It was divided into two parts, the first dedicated to the development of a questionnaire showing the highest sensitivity and specificity for $\mathrm{CV}$, which was then included in the second part of the study (part II), where the standard methodology for classification studies was used. ${ }^{13} 14$

\section{MATERIALS AND METHODS}

The study was proposed by GISC (Italian Study Group on Cryoglobulinemia). The experts agreed on four key points for the study development:

- Classification criteria are necessary for all the patients with $\mathrm{CV}$, either HCV-related or HCV-unrelated.

- The presence of serum cryoglobulins (either type I, II, III, or not typifiable) is an essential condition for the classification of $\mathrm{CV}$.

- The study protocol should be divided into two parts: part I, to develop a dedicated questionnaire for patients with $\mathrm{CV}$, and part II-that is, the formal study, to develop the classification criteria, using a standard methodology and including the questions selected in study part $\mathrm{I}^{13}$

- The core set of items for the classification of CV should include the dedicated questionnaire plus the presence of easily accessible clinical manifestations and laboratory tests. For this reason, histopathology, flow cytometry studies and novel laboratory biomarkers were excluded from the core set.

Agreement was also reached on the inclusion criteria for patients and controls, a dedicated paper chart, a glossary for the study and statistical analysis.

There was no financial support for the study. The final study protocol was developed by the coordinating centre.

\section{Part I}

Seventeen experts from 12 centres, experienced in the diagnosis and care of $\mathrm{CV}$, proposed a panel of 83 questions for patients with CV. Redundant questions were then deleted and among the 33 remaining questions only those considered useful by at least two-thirds of the experts were selected: they included five questions on purpura, four on peripheral nerve or muscular symptoms, two each on fatigue, articular involvement and oral or ocular 
Table 1 Questions included in study part I: questions selected by monovariate and multivariate analysis

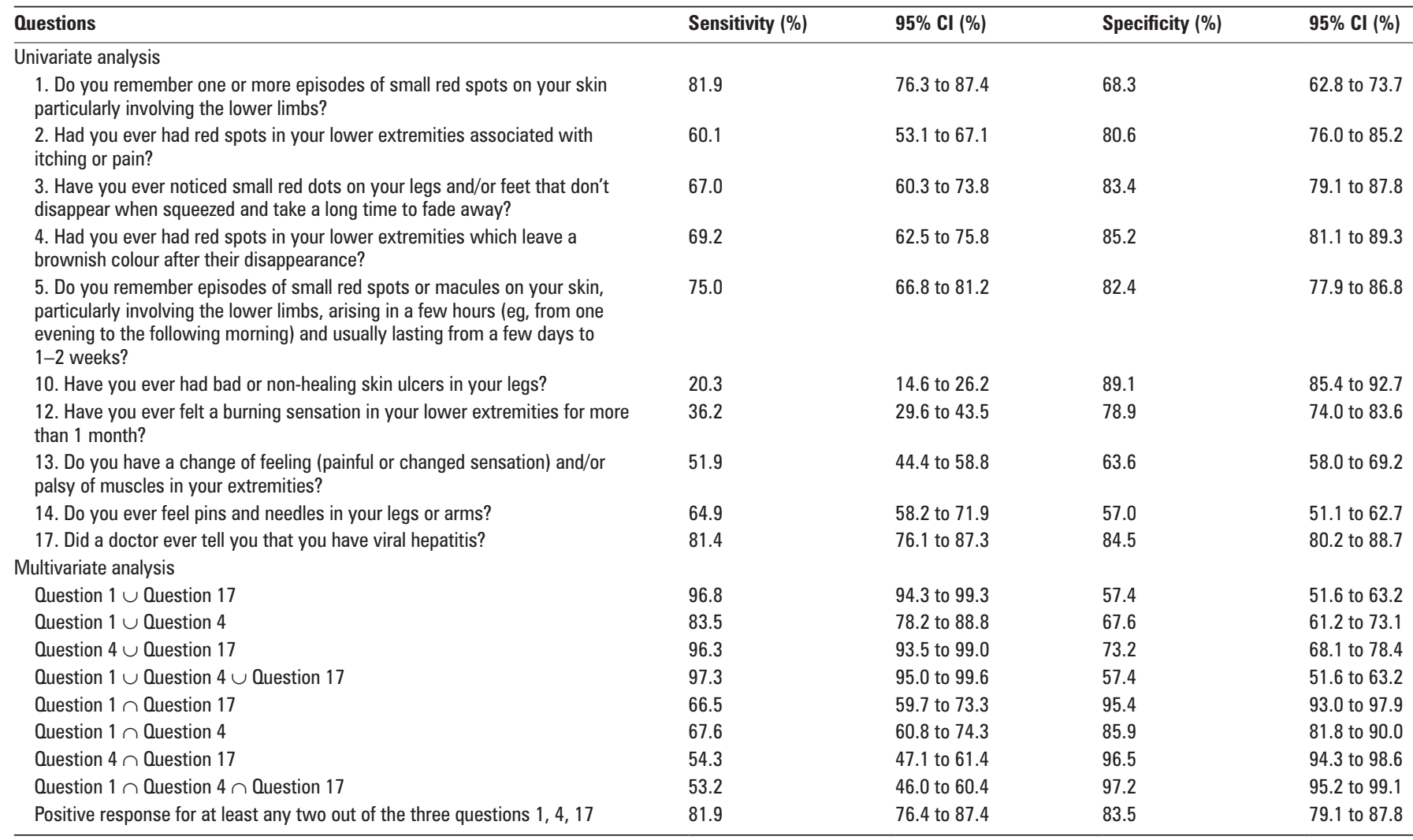

$\cup$, union; $\cap$, intersection.

dryness, and one each on leg skin ulcers and hepatitis virus infection (table 1).

A questionnaire consisting of the 17 selected questions was then sent to each centre, where it was translated into the local language and administered to 20 consecutive unselected patients with CV (HCV-related or HCV-unrelated), and to 30 consecutive, unselected controls without $\mathrm{CV}$ in care in the same centre for at least 1 year. The controls, with or without serum cryoglobulins, included 10 patients with systemic vasculitis other than CV, four HCV antibody and HCV-RNA-positive patients without CV, four patients with primary Sjögren's syndrome (SS), four with other connective tissue diseases, four with rheumatoid arthritis, polymyalgia rheumatica or seronegative spondyloarthritis, and four with any other condition where the differential diagnosis with CV was considered. The presence of cryoglobulins was assessed according to the local laboratory standard, based on reference methodologies, ${ }^{15}$ and confirmed by at least two positive tests at $\geq 12$ weeks' interval.

\section{Part II}

The second and formal part of the study was a cooperative study involving 16 centres, some of which were different from those involved in study part I. A dedicated paper chart was developed and included the following core set of items for classification:

1. a questionnaire for $\mathrm{CV}$ (with the questions selected in study part I);

2. data (89 items) on the pattern of organ involvement (present and past);

3. laboratory tests (28 items).

The classification of cases and controls as CV-positive and $\mathrm{CV}$-negative was based on the 'gold standard' of an expert clinician, who had never examined that case/control before. If CV had been diagnosed previously, the expert clinician had to redetermine the diagnosis based on his/her gold standard judgement.

Patients and controls included additional, consecutive and unselected cases and controls not enrolled in part I of the study, subdivided as follows into three groups $\mathrm{A}, \mathrm{B}$ and $\mathrm{C}$ :

- Group A: patients with CV, (20 per centre) essential or associated with other disorders (HCV-related or HCVunrelated), with type I, II, III or non-typifiable circulating cryoglobulins ${ }^{15}$ confirmed by at least two positive tests at $\geq 12$ weeks' interval.

- Group B: subjects (20 per centre) with serum cryoglobulins, but lacking a CV based on the gold standard judgement of the expert clinician (20 controls per centre), to try to answer the question "if a patient has positive serum cryoglobulins, when should a CV be classified?" For the inclusion of controls in this group, the minimum follow-up required was 1 year (ie, the expert did not notice any manifestations suggesting a $\mathrm{CV}$, during all the available history of the patient, with a minimum of a 12-month history needed).

- Group C: subjects without serum cryoglobulins (by at least two repeated tests during a follow-up of at least 1 year), but with clinical or laboratory features which can be observed in the course of CV (40 controls per centre), to try to answer the question "if a patient has some features which pose a differential diagnosis from $\mathrm{CV}$, even if negative for serum cryoglobulins by initial testing, when should a CV be suspected?"

Furthermore, a distinction in group $\mathrm{C}$ was made between patients with either systemic vasculitis (group C1: 20 controls per centre) or other diseases, which, however, should be distinguished from CV in clinical practice (group C2: 20 controls per 
centre). Patients in group $\mathrm{C}$ had a previous follow-up of at least 12 months to allow a definite diagnosis, other than CV.

The classification criteria for $\mathrm{CV}$ developed by comparing data in group A (CV) versus group B (positive serum cryoglobulins without $C V$ ) were then also tested by comparing group $\mathrm{A}$ versus group C (as a whole, or distinguishing C1 and C2).

All the patients and controls underwent a complete physical examination. The response to the selected questions, the clinical and laboratory core set data, plus instrumental tests and possibly additional investigations required by the clinician, were recorded in the chart. Chest x-ray and abdomen ultrasound examinations had to be performed in the 6 months before or at the time of the gold standard evaluation. If respiratory symptoms or abnormal thorax x-ray findings were present, a pulmonary function test, $\mathrm{CO}$ diffusion test and/or chest CT scanning had to be performed whenever possible, and electromyography was recommended if peripheral neuropathy was suspected.

All the data were entered and analysed by the coordinating centre of the study - namely, Rheumatology Clinic, Azienda OspedalieroUniversitaria 'S Maria della Misericordia', Udine, Italy.

\section{DATA ANALYSIS METHODS \\ Sample size}

Sample size estimation was derived from the accuracy of the estimation of the $95 \%$ CI (two-sided) for each sensitivity and specificity value.

\section{Part I of the study}

A sample size of 216 cases was necessary for a sensitivity of $90 \%$ with a precision of $\pm 4 \%$ at a two-sided confidence level of $95 \%$. With the same precision of $\pm 4 \%$ at a two-sided confidence level of $95 \%$, the sample size required to estimate a specificity of $85 \%$, was 306 controls. A higher sensitivity of the questionnaire was then presumed a priori.

A univariate analysis was carried out using $\chi^{2}$ test to identify the questions associated with CV. A stepwise multiple logistic regression was used to select the questions with greater accuracy to correctly classify patients and controls and then to select the best combination for classification. When different 'additive or multiplicative' combinations of questions were tested, the rule for classification was that 'for a given subject, at least $s$ of the $n$ questions were present'. If so, the subject was classified as having $\mathrm{CV}$.

\section{Part II of the study}

Since classification criteria aim to have a high specificity, together with a reasonable sensitivity, a sample size of 216 controls was calculated, estimating a specificity of $90 \%$ with a precision of $\pm 4 \%$ at a two-sided confidence level of $95 \%$, while the sample size to estimate a sensitivity of $85 \%$ with the same precision of $\pm 4 \%$ was 306 cases.

A univariate analysis was carried out to identify the questions, the clinical features and laboratory tests associated with CV. The items significantly associated with CV by univariate analysis were then analysed by a stepwise multiple logistic regression. This analysis was performed separately for questions, clinical features and laboratory tests.

Then, different 'additive or multiplicative' combinations of items were tested for sensitivity and specificity. The rule was that 'for a given subject, at least $s$ of the $n$ items were present'.

Finally, with the same analysis, the best combination of the three sets of items-that is, the set of questions, the set of clinical features and the set of laboratory tests, was analysed to determine the final classification criteria.

A tree-based model was also used as an alternative method to correctly classify patients and controls. ${ }^{67} 13$ The model is fitted by binary recursive partitioning whereby a dataset is successively split into increasingly homogeneous subsets until it is infeasible to continue.

\section{RESULTS}

\section{Part I of the study}

Of the 12 selected centres, 10 completed this part of the study, for a total of 484 questionnaires available. The results of the questionnaires were received in electronic form by the coordinating centre and were rechecked with the corresponding paper charts. Of the 484 questionnaires, 472 were used for statistical analysis (the remaining 12 were incomplete).

Cases comprised 188 subjects (68.1\% women), with a mean age of $59.18 \pm 15.14$ years; controls were 284 subjects $(72.5 \%$ women), with a mean age of $53.76 \pm 15.24$ years.

Univariate analysis identified questions 1, 2, 3, 4, 5, 10, 12, 13, 14 and 17 as associated with CV, but the logistic model showed that only questions 1,4 and 17 contributed significantly to the predictability of the disease. The sensitivity and specificity of each question, and of all the possible combinations of positive answers to questions 1, 4 and 17, are shown in table 1.

The best rule for classification was a positive response to at least two of the three questions 1, 4 and 17, with a sensitivity of $81.9 \%$ (estimated with a precision of $5.5 \%$ ) and a specificity of $83.5 \%$ (with a precision of $4.4 \%$ ) for CV. In addition to questions 1, 4 and 17, questions 10,12 and 14 were also included in part II of the study, since the experts considered that repeated investigation was worthwhile for skin ulcers and peripheral neuropathy, based on the results of univariate analysis.

\section{Part II of the study}

Overall, 925 charts were received by the coordinating centre, which performed the electronic data entry, checked three times before data analysis.

Group A included 272 patients with CV (73.5\% women), with a mean age $62.8 \pm 11.8$ years. Group B included 228 controls with serum cryoglobulins but lacking a CV (68.4\% women), mean age $58.4 \pm 15.4$ years. Group $\mathrm{C}$ included 425 controls lacking serum cryoglobulins though with clinical or laboratory features which can be observed in the course of CV (72.2\% women), mean age $56.0 \pm 16.8$ years, divided into 173 controls in group C1 (systemic vasculitis other than CV: $63.6 \%$ women, mean age $58.3 \pm 17.8$ years) and 252 controls in group C2 (other diseases: $78.2 \%$ women, mean age $54.5 \pm 16.0$ years). Cases in group C1 comprised 55 antineutrophil cytoplasmic antibody-associated vasculitis (Wegener's granulomatosis, Churg-Strauss syndrome, micropolyarteritis), 47 giant cell arteritis, 15 isolated cutaneous vasculitis, 14 Schönlein-Henoch purpura, 12 panarteritis nodosa, 9 Takayasu arteritis, 6 Beçhet disease, 2 Goodpasture's syndrome, 13 other vasculitis. Cases in group C2 were 52 SS, 36 systemic sclerosis, 35 systemic lupus erythematosus, 30 rheumatoid arthritis, 11 polymyositis or dermatomyositis, 10 spondyloarthritis, 7 polymyalgia rheumatica, 3 antiphospholipid syndrome, 3 undifferentiated connective tissue disease and 1 mixed connective tissue disease, and 64 with other diseases which should be distinguished from CV in clinical practice (including 31 chronic HCV infection, 20 haematological malignancies and various other conditions such as sarcoidosis, 
Table 2 Composition of groups of patients in study part II

\begin{tabular}{llllll}
\hline & Group A & Group B & Group C & Group C1 & Group C2 \\
\hline No of patients & 272 & 228 & 425 & 173 & 252 \\
Mean age (SD) & $62.8(11.8)$ & $58.4(15.4)$ & $56.0(16.8)$ & $58.3(17.8)$ & $54.5(16.0)$ \\
Sex (M/F) & $72 / 200$ & $72 / 156$ & $118 / 307$ & $63 / 110$ & $55 / 197$ \\
Cryo type I (\%) & $5(1.8)$ & $1(0.4)$ & - & - & - \\
Cryo type II (\%) & $194(71.3)$ & $40(17.5)$ & - & - & - \\
Cryo type III (\%) & $32(11.8)$ & $110(48.2)$ & - & - & - \\
Cryo not typifiable (\%) & $41(15.1)$ & $77(33.8)$ & - & - & - \\
Rheumatoid factor positivity (\%) & 85.8 & 46.3 & 25.2 & 12.4 & 33.9 \\
Low C4 (\%) & 69.6 & 25.5 & 9.2 & 1.9 & 14.4 \\
M component (\%) & 82.7 & 27.2 & 3.7 & 2.8 & 4.4 \\
Truly essential cryo (\%) & $9(3.3)$ & $7(3.1)$ & - & - & - \\
HCV infection (\%)* & $230(84.6)$ & $153(67.1)$ & $31(7.3)$ & - & $31(12.3)$ \\
SS (\%) & $39(14.4)$ & $34(14.9)$ & $52(12.2)$ & - & $52(20.6)$ \\
Other CTD s ${ }^{\dagger}(\%)$ & $5(1.8)$ & $30(13.2)$ & $89(20.9)$ & - & $89(35.3)$ \\
Other vasculitis ${ }^{\ddagger}(\%)$ & - & - & $173(40.7)$ & $173(100)$ & - \\
Other diseases ${ }^{\ddagger}(\%)$ & $10(3.7)$ & $8(3.5)$ & $80(18.8)$ & - & $80(31.7)$ \\
\hline
\end{tabular}

${ }^{*} \mathrm{HCV}$ infection could be also concomitant with SS or with other connective tissue diseases.

${ }^{\dagger}$ Systemic lupus erythematosus, systemic sclerosis, poly/dermatomyositis, undifferentiated connective tissue disease, mixed connective tissue disease, antiphospholipid syndrome.

${ }^{\ddagger}$ In groups $A$ and $B$, positivity for the hepatitis B virus surface antigen was noticed in 10 and in eight cases, respectively; see text for other diseases included in group $\mathrm{C}$, to be differentiated from CV.

CTDs, connective tissue diseases; CV, cryoglobulinaemic vasculitis; F, female; HCV, hepatitis C virus; M, male; SS, Sjögren's syndrome.

erythema nodosum, idiopathic thrombocytopenic purpura, palindromic rheumatism, venous insufficiency in lower limbs). The group distribution is reported in table 2.

Overall, about 89000 clinical items and 18000 laboratory items were filed.

\section{Comparison of group A with group B (cryoglobulinaemic syndrome versus serum cryoglobulinaemia without syndrome) and development of the classification criteria}

Questionnaire validation

All the six questions included in part II of the study were significantly associated with $\mathrm{CV}$ by the univariate analysis, but only questions 1, 4 and 17 (table 3 ) were also selected by the multivariate analysis. This fully replicated the results of part I of the study, even if the controls were selected differently in part II.

When different 'additive or multiplicative' combinations were tested on the three questions, the best criterion was 'at least two out of the three questions', giving a sensitivity of $83.8 \%, 95 \%$ CI $(79.4 \%$ to $88.2 \%)$ and a specificity of $93.8 \%, 95 \%$ CI $(90.7 \%$ to $97.0 \%$ ) for CV. The sensitivity and specificity of this combination were higher in study part II than in study part I $(81.9 \%$ and $83.5 \%$, respectively). Thus, these results of part II of the study validated a three-question-based questionnaire for $\mathrm{CV}$ developed by study part I.

\section{Clinical features}

Constitutional symptoms fatigue, low-grade fever $\left(37-37.9^{\circ} \mathrm{C}\right.$ for more than 10 days) or fever $\left(\geq 38^{\circ} \mathrm{C}\right)$, or fibromyalgia, ${ }^{16}$ articular involvement (arthralgias or arthritis), vascular involvement (purpura, skin ulcers, necrotic skin lesions or necrotising vasculitis biopsy-proven, Raynaud's phenomenon, or hyperviscosity syndrome according to clinical features and plasma viscosity test) and neurological involvement (peripheral neuropathy confirmed by electromyography or confirmed by a neurologist in electromyography-negative cases, cranial nerve involvement confirmed by a neurologist, or central nervous system vasculitic involvement documented by imaging) were the clinical items selected by the multivariate analysis, to establish CV in patients with positive serum cryoglobulins. Their sensitivity and specificity values by the univariate analysis are shown in table 3.

The criterion of 'at least three of the four items' gave a sensitivity of $70.2 \%, 95 \%$ CI $(64.7 \%$ to $75.8 \%)$ and a specificity of $84.5 \%, 95 \%$ CI $(79.5 \%$ to $89.4 \%)$ for CV, while the criterion of 'at least two of the four items' gave a higher sensitivity $90.1 \%$, $95 \%$ CI $86.4 \%$ to $93.7 \%$ ) but a much lower specificity $(65.5 \%$, $95 \%$ CI $59.0 \%$ to $72.1 \%$ ) for CV.

\section{Laboratory features}

Reduced C4 levels, positive RF and the presence of serum M component (present in type I or II cryoglobulins, or detected by serum protein electrophoresis and immunofixation) were the laboratory items selected by the multivariate analysis, to establish a CV in patients with positive serum cryoglobulins. Sensitivity and specificity in the univariate analysis are shown in table 3 . The criterion of 'at least two out of the three features' gave a sensitivity of $84.2 \%, 95 \%$ CI $(79.4 \%$ to $89.0 \%)$ and a specificity of $79.6 \%, 95 \%$ CI $(73.4 \%$ to $85.9 \%)$ for $\mathrm{CV}$.

\section{Classification criteria for the cryoglobulinaemic syndrome}

By analysing the best combination of the three sets of itemsthat is, the set of questions ( $\geq 2$ of 3 ), the set of clinical features ( $\geq 3$ of 4 ), and the set of laboratory tests ( $\geq 2$ of 3 ), to determine the final classification criteria, the criterion of at least two out of three questionnaire, clinical and laboratory sets of items (questionnaire + clinical; questionnaire + laboratory; or clinical + laboratory) provided a sensitivity of $88.5 \%, 95 \%$ CI $(84.3 \%$ to $92.8 \%$ ) with a precision of $\pm 4.3 \%$, and a specificity of $93.6 \%$, $95 \%$ CI $(89.5 \%$ to $97.7 \%)$ with a precision of $\pm 4.1 \%$ for CV (table 4 and figure 1).

Other combinations, including the validated questions plus the positivity of lower or higher numbers of clinical or laboratory items, provided worse results, and the cost of a higher sensitivity was a low specificity for classification purposes.

A tree-based model for predicting $\mathrm{CV}$, derived by applying the criterion of 'at least two out of' the three questionnaire (1), clinical (2) and laboratory (3) features, to 272 patients with CV and 228 controls with serum cryoglobulins but lacking a CV is shown in figure 2. The probability of CV for patients with positive 
Table 3 Results of group A vs group B analyses: sensitivity and specificity of the different questions, clinical features and laboratory features included in the anamnestic, clinical and laboratory item, respectively

\begin{tabular}{|c|c|c|c|c|}
\hline & Sensitivity (\%) & $95 \% \mathrm{Cl}(\%)$ & Specificity (\%) & $95 \% \mathrm{Cl}(\%)$ \\
\hline \multicolumn{5}{|l|}{ Questionnaire item } \\
\hline $\begin{array}{l}1 \rightarrow 1 \text { Do you remember one or more episodes of small red spots on } \\
\text { your skin particularly involving the lower limbs? }\end{array}$ & 83.5 & 79.0 to 87.9 & 89.9 & 85.6 to 93.8 \\
\hline $\begin{array}{l}4 \rightarrow 2 \text { Have you ever had red spots on your lower extremities which } \\
\text { leave a brownish colour after their disappearance? }\end{array}$ & 74.3 & 69.0 to 79.5 & 94.7 & 91.8 to 97.6 \\
\hline $17 \rightarrow 3$ Has a doctor ever told you that you have viral hepatitis? & 83.1 & 78.6 to 87.6 & 38.6 & 32.2 to 44.9 \\
\hline \multicolumn{5}{|l|}{ Clinical item } \\
\hline Constitutional symptoms & 81.5 & 76.9 to 86.2 & 56.8 & 50.3 to 63.3 \\
\hline Articular involvement & 63.0 & 57.2 to 68.7 & 57.0 & 50.6 to 63.5 \\
\hline Vascular involvement & 87.9 & 84.0 to 91.8 & 78.5 & 73.1 to 83.9 \\
\hline Neurological involvement & 64.4 & 58.6 to 70.2 & 86.5 & 81.8 to 91.2 \\
\hline \multicolumn{5}{|l|}{ Laboratory item } \\
\hline Low serum C4 & 69.6 & 64.0 to 75.2 & 74.5 & 68.3 to 80.6 \\
\hline Rheumatoid factor (RF) positivity & 85.8 & 81.5 to 90.1 & 53.7 & 46.8 to 60.7 \\
\hline Presence of serum M component & 83.1 & 78.4 to 87.8 & 72.8 & 66.2 to 79.3 \\
\hline \multicolumn{5}{|l|}{ Group A vs group $B$} \\
\hline 'At least 2 out of the 3 questions' & 83.8 & 79.4 to 88.2 & 93.8 & 90.7 to 97.0 \\
\hline $\begin{array}{l}\text { 'At least } 3 \text { out of the } 4 \text { features' among constitutional symptoms, } \\
\text { articular, vascular or neurological involvement }\end{array}$ & 70.2 & 64.7 to 75.8 & 84.5 & 79.5 to 89.4 \\
\hline $\begin{array}{l}\text { 'At least } 2 \text { out of the } 3 \text { features' among serum C4 levels, RFs and } \\
\text { serum M component }\end{array}$ & 84.2 & 79.4 to 89.0 & 79.6 & 73.4 to 85.9 \\
\hline
\end{tabular}

Table 4 Results of group A vs group C, and of group A vs group C1 analyses: sensitivity and specificity of the different questions, clinical features and laboratory features included in the anamnestic, clinical and laboratory item, respectively

\begin{tabular}{|c|c|c|c|c|}
\hline & Sensitivity (\%) & $95 \% \mathrm{Cl}(\%)$ & Specificity (\%) & $95 \% \mathrm{Cl}(\%)$ \\
\hline \multicolumn{5}{|l|}{ Group A vs group C } \\
\hline 'At least 2 out of the 3 questions' & 83.8 & 79.4 to 88.2 & 90.6 & 87.8 to 93.4 \\
\hline $\begin{array}{l}\text { 'At least } 3 \text { out of the } 4 \text { features' among constitutional symptoms, } \\
\text { articular, vascular or neurological involvement }\end{array}$ & 70.2 & 64.7 to 75.8 & 68.7 & 64.2 to 73.3 \\
\hline $\begin{array}{l}\text { 'At least } 2 \text { out of the } 3 \text { features' among serum C4 levels, rheumatoid } \\
\text { factors (RFs) and serum M component }\end{array}$ & 84.2 & 79.4 to 89.0 & 97.7 & 95.7 to 99.7 \\
\hline \multicolumn{5}{|l|}{ Group A vs group C1 (systemic vasculitis) } \\
\hline 'At least 2 out of the 3 questions' & 83.8 & 79.4 to 88.2 & 85.5 & 80.3 to 90.8 \\
\hline $\begin{array}{l}\text { 'At least } 3 \text { out of the } 4 \text { features' among constitutional symptoms, } \\
\text { articular, vascular or neurological involvement }\end{array}$ & 70.2 & 64.7 to 75.8 & 53.0 & 45.4 to 60.7 \\
\hline $\begin{array}{l}\text { 'At least } 2 \text { out of the } 3 \text { features' among serum C4 levels, RFs and } \\
\text { serum M component }\end{array}$ & 84.2 & 79.4 to 89.0 & 100 & \\
\hline
\end{tabular}

questionnaire and laboratory criteria was $99.4 \%$. The final probability of CV provided by the tree-based model ("at least two out of the three questions' $\rightarrow$ 'at least two out of the three laboratory features' $\rightarrow$ 'at least three out of the four clinical features') was $100 \%$. Similarly, the probability of lacking CV for patients who did not satisfy the questionnaire and laboratory criteria was $92.1 \%$, and the final probability of lacking CV provided by the tree-based model ('less than two out of the three questions' $\rightarrow$ 'less than two out of the three laboratory features' $\rightarrow$ 'less than three out of the four clinical features') was $94.6 \%$ (figure 2).

\section{Comparison of group A with group C (cryoglobulinaemic syndrome versus other diseases which may mimic the syndrome)}

The classification criteria developed in comparing group A with group B were applied for the comparison of group A with group C, to verify whether the classification criteria may also be useful to suspect a CV in patients cryoglobulin-negative by initial testing, though showing clinical or laboratory features suggestive of CV.

The results of statistical analyses for questions, clinical manifestations and laboratory features associated with CV are shown in table 4.
The criteria provided satisfactory results for sensitivity $88.5 \%$ $95 \%$ CI (84.3\% to $92.8 \%)$ and specificity $97.0 \%, 95 \%$ CI $(94.6 \%$ to $99.4 \%$ ).

When further analysing group A versus group C1 (including only patients with systemic vasculitis other than $\mathrm{CV}$ ), similar results were noticed (table 4).

The final criteria provided a sensitivity of $88.5 \%, 95 \% \mathrm{CI}$ $(84.3 \%$ to $92.8 \%)$ and a specificity of $95.4 \%, 95 \%$ CI $(90.9 \%$ to $99.8 \%$ ). Thus, if a systemic vasculitis is suspected, positive CV criteria, even in the absence of positive serum cryoglobulins by initial testing, should warn the clinician of a possible CV, and repeated cryoglobulin testing is mandatory.

\section{DISCUSSION}

The preliminary classification criteria for the CV are presentedthe result of a multicentre European study. Classification criteria for CV developed by a standardised methodology were lacking, though they are crucial in clinical practice, scientific communication and epidemiological surveys. A methodology similar to that employed for the classification criteria for SS was used..$^{13} 14$ This led, as a first step, to the development of a dedicated questionnaire. The questionnaire was then validated and the set of classification criteria for CV was defined, also including easily 
(i) Questionnaire item: at least two out of the following

- Do you remember one or more episodes of small red spots on your skin, particularly involving the lower limbs?

- Have you ever had red spots on your lower extremities which leave a brownish color after their disappearance?

- Has a doctor ever told you that you have viral hepatitis?

(ii) Clinical item : at least three out of the following four (present or past)*

- Constitutional symptoms Fatigue

Low grade fever $\left(37-37.9^{\circ} \mathrm{C},>10\right.$ days, no cause $)$

Fever $\left(>38^{\circ} \mathrm{C}\right.$, no cause)

Fibromyalgia

- Articular involvement Arthralgias

Arthritis

- Vascular involvement Purpura

Skin ulcers

Necrotising vasculitis

Hyperviscosity syndrome

Raynaud's phenomenon

- Neurologic involvement Peripheral neuropathy

Cranial nerve involvement

Vasculitic CNS involvement

iii. Laboratory item : at least two out of the following three (present)

- Reduced serum C4

- Positive serum rheumatoid factor

- Positive serum M component

*See text for details

Figure 1 Preliminary classification criteria for the cryoglobulinaemic vasculitis.

accessible clinical and laboratory items. The criteria showed a high specificity and a good sensitivity for CV. In addition, although this was not the main purpose of the study, the same criteria were also investigated and proved useful in classifying $\mathrm{CV}$ in patients with negative serum cryoglobulins by initial laboratory testing.

A uniform set of classification criteria for CV was needed. Previous criteria were non-universal, employed by the experts who had proposed them, and lacked appropriate statistical support. ${ }^{9-12}$ Guidelines for the management of CV have been recently proposed, ${ }^{8}$ but uniform criteria to classify $\mathrm{CV}$ were not available at that time.

This study, proposed by GISC ${ }^{9}$ included experts from five European countries and with different backgrounds. The methodology used, based on a gold standard preliminary diagnosis of CV, meets the current standards for classification criteria in disorders characterised by the lack of a single, reliable diagnostic test. For the final set of classification criteria, a combination showing a high specificity was needed. This goal was achieved (specificity of 93.6\%), and a good sensitivity (88.5\%) for CV was maintained, with a good precision for both. The questions selected in part I of the study proved relevant for classification in study part II, by comparing patients with cryoglobulins and CV (group A) with patients with cryoglobulins but without CV (group B).

The results of this study confirm that in CV, as in other autoimmune diseases, classification can be made by integrating different questionnaire, clinical and laboratory data. Of note, none of the three sets of items comprising the CV classification criteria herein developed (positive answer to $\geq 2 / 3$ validated questions, presence of $\geq 3 / 4$ clinical features, or presence of $\geq 2 / 3$ laboratory abnormalities) is absolutely needed for classification itself, since classification is possible with positivity for any two of the three questionnaire, clinical or laboratory items. This is an advantage for patients with CV with difficulties in completing a questionnaire, for patients with clinical features less common in CV (eg, abdominal vasculitis, serositis, glomerulonephritis, etc), or where laboratory data are uncertain for any reason.

Although this study shows that no fixed combination of tests can be used to classify CV, the classification tree suggests a sequence of tests to be followed in the diagnostic approach to the disease. A validated three-item questionnaire is useful for initial evaluation of the patient: if a patient has positive serum cryoglobulins and is also positive for the questionnaire criterion, the probability of $\mathrm{CV}$ is $94.4 \%$, with a further increase to $99.4 \%$ if the laboratory criterion is also positive. This approach may be particularly useful if CV is highly suspected by doctors without extensive experience of this disease, before the evaluation of the specialist. Conversely, if a patient with serum cryoglobulins is negative for the questionnaire criterion and is also lacking the CV laboratory features included in the laboratory criterion, CV can be excluded with a probability of $92.1 \%$.

One main criticism of these criteria is that they cannot be applied to patients with CV with negative serum cryoglobulins. This point was thoroughly discussed in study planning, where positive serum cryoglobulins were finally designated as an essential condition for CV classification. For classification purposes it was established that it is not possible to classify the CV if the characterising feature-that is, positive serum cryoglobulins, is not demonstrated. However, it was agreed that if cryoglobulinaemia is absent at a definite evaluation point, but has been well documented in the past (eg, before antiviral or anti-CD20 treatment), cryoglobulin positivity rather than negativity must be considered.

There is a subset of CV in which serum cryoglobulins may appear to be negative by initial laboratory testing for various reasons, such as low cryoglobulin levels, problems in blood sample handling or testing, or owing to tissue deposition rather than blood circulation of immune complexes. Such patients deserve a careful follow-up if CV is suspected, and must undergo repeat cryoglobulin determination, while excluding other diseases. This led to an extensive expert discussion on 


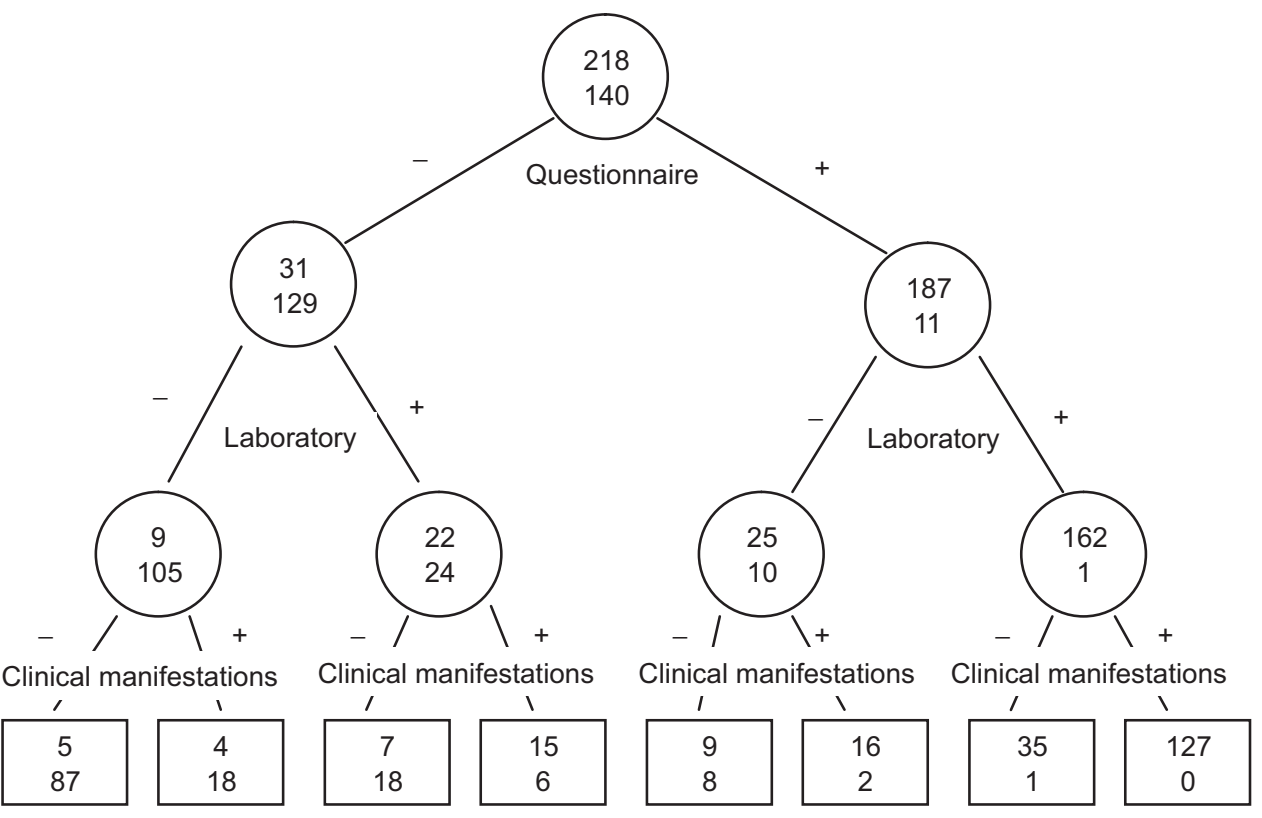

Figure 2 Schematic representation of the classification tree for the cryoglobulinaemic vasculitis (CV). Within each circle and box is the number of the patients with CV according to the 'gold standard' (upper value) and the number of controls without CV according to the gold standard (lower value). Interior nodes are denoted by circles and terminal nodes by boxes. The criterion used in each node of the tree to discriminate between cases and controls is reported beneath the circles. Details of the three-item criterion set, questionnaire, clinical and laboratory, are given in Figure 1; they were assessed by the binary recursive partitioning method. Only those subjects for whom the entire three-item criterion set was available were included in this procedure (358 subjects: 218 patients with CV and 140 controls). The sequential approach selected the questionnaire item as the first node of the tree, which divided the 358 observations into groups of 160 and 198 individuals, with a probability of CV of $19.4 \%$ (less than two out of the three questions) and $94.4 \%$ (at least two out of the three questions), respectively. The latter group, with greater probability of CV, was then partitioned into groups of 35 and 163 individuals, depending on whether the item 'at least two out of the three laboratory features' was satisfied. The respective probabilities of CV for these groups were $71.4 \%$ and $99.4 \%$, respectively. Both these groups were further subdivided by the item 'at least three out of the four clinical features', and the latter one, with greater probability of CV (99.4\%) was partitioned into groups of 36 and 127 individuals with probability of CV of $97.2 \%$ and $100 \%$, respectively. Then, the final probability of CV provided by the tree-based model ('at least two out of the three questions' $\rightarrow$ 'at least two out of the three laboratory features' $\rightarrow$ 'at least three out of the four clinical features') was $100 \%$, based on 127 individuals. Similarly, the final probability of lacking a CV, beginning from the first node of the tree, was $80.6 \%$ (less than two out of the three questions) and $5.6 \%$ (at least two out of the three questions), respectively. The former group, with greater probability of lacking a CV, was then partitioned into groups of 114 and 46 individuals depending on whether the item 'at least two out of the three laboratory features' was or was not satisfied. The probabilities of lacking a CV for these groups were $92.1 \%$ (less than two out of the three laboratory features) and $52.2 \%$ (at least two out of the three laboratory features). Both these groups were further subdivided by the item 'at least three out of the four clinical features', and the former one, with greater probability of lacking a CV (92.1\%) was partitioned into groups of 92 (less than three out of the four clinical features) and 22 (at least three out of the four clinical features) individuals with probability of lacking a CV of $94.6 \%$ and $81.8 \%$, respectively. Then, the final probability of lacking CV provided by the tree-based model ('less than two out of the three questions' $\rightarrow$ 'less than two out of the three laboratory features' $\rightarrow$ 'less than three out of the four clinical features') was $94.6 \%$, based on 92 individuals.

classification problems in these cases, and the study was finally designed in an attempt to clarify whether classification criteria (developed by comparing cryoglobulin-positive patients with or without $\mathrm{CV}$ ) might be of some relevance for $\mathrm{CV}$ diagnosis when serum cryoglobulins appear to be absent. By comparing data in group A with those in group C, it was shown that the classification criteria were useful in such cases, since positive criteria were rarely present in diseases that may mimic $\mathrm{CV}$, including a group of patients (C1) with systemic vasculitis other than $\mathrm{CV}$. Then, if $\mathrm{CV}$ is suspected, positive classification criteria for $\mathrm{CV}$ in that patient, even in the absence of detectable cryoglobulins by initial testing, warn the clinician to repeat the cryoglobulin determination, and classification of CV may be possible in the follow-up. This part of the study supported the clinical practice of repeating cryoglobulin laboratory determination if CV is highly suspected on clinical grounds, and the relevance of sample handling and high-quality laboratory referral for CV assessment.

Second, an extremely small number of patients with CV and type I cryoglobulins were included in this study, and this in part reflects the rarity of this subset, which usually occurs in the course of an overt B-cell lymphoproliferative disorder. All except one of these cases were correctly classified with the criteria developed (data not shown). However, these criteria cannot be formally proposed for patients with type I cryoglobulinaemia, and the experts agreed that this patient subset should be expanded in subsequent validation studies.

Furthermore, the distinction between classification and diagnostic criteria should be emphasised. Classification criteria are constructed mainly for investigation and epidemiological purposes, and aim at a higher specificity. ${ }^{67}$ By contrast, the diagnosis of a disease can be formulated by the expert clinician also in patients lacking the classification criteria for that disease, and prompt diagnosis and treatment decisions are certainly more relevant in clinical practice. ${ }^{13}{ }^{17-19}$ We would at present discourage the use of these classification criteria for diagnostic purposes, and additional data analyses are under way.

In conclusion, preliminary classification criteria for $\mathrm{CV}$ have been developed by a cooperative study using a standardised methodology in a large number of real cases. They need a 
formal validation, possibly involving experts from a larger number of countries. ${ }^{20} 21$ In addition, they should be further tested in HCV-related versus HCV-unrelated CV. These studies, as well as more descriptive analyses and subanalyses, are continuing or have been planned.

Acknowledgements The authors are grateful to Dr Leonard Calabrese, Cleveland, Ohio, USA, for his comments in manuscript preparation, and to Dr Alessandra Della Rossa, Pisa, Italy; Dr Sara Salvin, Udine, Italy and Dr Marta Maset, Udine, Italy, for their contribution to patient recruitment and data collection.

Patient consent Obtained.

Ethics approval This study was conducted with the approval of the Udine ethics committee.

Provenance and peer review Not commissioned; externally peer reviewed.

Author affiliations ${ }^{1}$ Rheumatology Clinic, Department of Medical and Biological Sciences, Azienda Ospedaliero-Universitaria S Maria della Misericordia, Udine, Italy ${ }^{2}$ Chair of Statistics, DIRMM, University of Udine, Udine, Italy

${ }^{3}$ Internal Medicine Unit, Saronno Hospital, Azienda Ospedaliera di Busto Arsizio, Saronno Varese, Italy

${ }^{4}$ Internal Medicine, Università Politecnica delle Marche, Ancona, Italy

${ }^{5}$ Department of Pathophysiology, Medical School of Athens, Athens, Greece

${ }^{6}$ Rheumatology Unit, University of Modena and Reggio Emilia, Italy

${ }^{7}$ Rheumatology Division, Catholic University of the Sacred Heart, School of Medicine, Rome, Italy

${ }^{8}$ Laboratorio de Enfermedades Autoinmunes Josep Font, IDIBAPS, Hospital Clínic, Barcelona, Spain

${ }^{9}$ Department of Hematology, Medical School, University of Athens, Athens, Greece

${ }^{10}$ Department of Clinical Medicine, University of Bologna, S Orsola-Malpighi Hospital, Bologna, Italy

${ }^{11}$ Section of Internal Medicine and Clinical Oncology, Department of Biomedical Sciences and Human Oncology, University of Bari, Bari, Italy

${ }^{12}$ Centre de références maladies auto-immunes, Service de médecine interne II, Hôpital Pitié-Salpêtrière, Paris, France

${ }^{13}$ Department of Rheumatology, University Medical Centre, Ljubljana, Slovenia

${ }^{14}$ Rheumatology Unit, Department of Internal Medicine, University of Pisa, Pisa, Italy

${ }^{15}$ Internal Medicine Unit, Policlinico San Marco, Bergamo, Italy

${ }^{16}$ Center for Systemic Manifestations of Hepatitis Viruses (MaSVE), Department of Internal Medicine, University of Florence, Florence, Italy

${ }^{17}$ Rheumatology Unit, M Scarlato Hospital, Scafati, Salerno, Italy

${ }^{18}$ Department of Internal Medicine, Pordenone General Hospital, Pordenone, Italy

${ }^{19}$ Internal Medicine, Donizetti Hospital, Monza, Italy

${ }^{20}$ Department of Rheumatology, Erasme University Hospital, Brussels, Belgium

${ }^{21}$ Institute of Immunology, University of Kiel, Kiel, Germany

${ }^{22}$ Istituto di Malattie Infettive e Tropicali, Università di Milano c/0 Ospedale L Sacco,

Milan, Italy

\section{REFERENCES}

1. Meltzer M, Franklin EC. Cryoglobulinemia - a study of twenty-nine patients. I. $\lg \mathrm{G}$ and $\operatorname{lgM}$ cryoglobulins and factors affecting cryoprecipitability. Am J Med 1966:40:828-36

2. Gorevic PD, Frangione B. Mixed cryoglobulinemia cross-reactive idiotypes: implications for the relationship of MC to rheumatic and lymphoproliferative diseases. Semin Hematol 1991;28:79-94.
3. De Vita S, De Re V, Gasparotto D, et al. Oligoclonal non-neoplastic B cell expansion is the key feature of type II mixed cryoglobulinemia: clinical and molecular findings do not support a bone marrow pathologic diagnosis of indolent B cell lymphoma. Arthritis Rheum 2000;43:94-102.

4. Ferri C, Greco F, Longombardo G, et al. Antibodies to hepatitis $\mathrm{C}$ virus in patients with mixed cryoglobulinemia. Arthritis Rheum 1991;34:1606-10.

5. Agnello V, Chung RT, Kaplan LM. A role for hepatitis C virus infection in type II cryoglobulinemia. N Engl J Med 1992;327:1490-5.

6. Johnson SR, Goek ON, Singh-Grewal D, et al. Classification criteria in rheumatic diseases: a review of methodologic properties. Arthritis Rheum 2007;57:1119-33.

7. Dougados M, Gossec L. Classification criteria for rheumatic diseases: why and how? Arthritis Rheum 2007;57:1112-15.

8. Mukhtyar C, Guillevin L, Cid MC, et al.; European Vasculitis Study Group. EULAR recommendations for the management of primary small and medium vessel vasculitis. Ann Rheum Dis 2009;68:310-17.

9. Invernizzi F, Pietrogrande M, Sagramoso B. Classification of the cryoglobulinemic syndrome. Clin Exp Rheumatol 1995;13(Suppl 13):S123-8.

10. Ferri C, Zignego AL, Pileri SA. Cryoglobulins. J Clin Pathol 2002;55:4-13.

11. Landau DA, Rosenzwajg M, Saadoun D, et al. Correlation of clinical and virologic responses to antiviral treatment and regulatory $T$ cell evolution in patients with hepatitis $C$ virus-induced mixed cryoglobulinemia vasculitis. Arthritis Rheum 2008;58:2897-907.

12. Kaplanski G, Maisonobe T, Marin V, et al. Vascular cell adhesion molecule-1 (VCAM-1) plays a central role in the pathogenesis of severe forms of vasculitis due to hepatitis C-associated mixed cryoglobulinemia. J Hepatol 2005;42:334-40.

13. Vitali C, Bombardieri S, Moutsopoulos HM, et al. Preliminary criteria for the classification of Sjögren's syndrome. Results of a prospective concerted action supported by the European Community. Arthritis Rheum 1993;36:340-7.

14. Lamprecht $\mathbf{P}$. [The cryoglobulinemic syndrome - report from the workshop on classification and on the 10th conference of the Italian Society for the treatment of Cryoglobulinemia, Modena, 29 Sept., 2003]. Z Rheumatol 2004;63:235-8.

15. Brouet JC, Clauvel JP, Danon F, et al. Biologic and clinical significance of cryoglobulins. A report of 86 cases. Am J Med 1974;57:775-88.

16. Wolfe F, Smythe HA, Yunus MB, et al. The American College of Rheumatology 1990 Criteria for the Classification of Fibromyalgia. Report of the Multicenter Criteria Committee. Arthritis Rheum 1990;33:160-72.

17. Hunder GG, Arend WP, Bloch DA, et al. The American College of Rheumatology 1990 criteria for the classification of vasculitis. Introduction. Arthritis Rheum 1990;33:1065-7.

18. Calabrese LH, Michel BA, Bloch DA, et al. The American College of Rheumatology 1990 criteria for the classification of hypersensitivity vasculitis. Arthritis Rheum 1990;33:1108-13.

19. Hunder GG, Bloch DA, Michel BA, et al. The American College of Rheumatology 1990 criteria for the classification of giant cell arteritis. Arthritis Rheum 1990;33:1122-8

20. Vitali C, Bombardieri S, Moutsopoulos HM, et al. Assessment of the European classification criteria for Sjögren's syndrome in a series of clinically defined cases: results of a prospective multicentre study. The European Study Group on Diagnostic Criteria for Sjögren's Syndrome. Ann Rheum Dis 1996;55:116-21.

21. Vitali C, Bombardieri S, Jonsson R, et al.; European Study Group on Classification Criteria for Sjögren's Syndrome. Classification criteria for Sjögren's syndrome: a revised version of the European criteria proposed by the American-European Consensus Group. Ann Rheum Dis 2002:61:554-8. 